\title{
A review of nasal polyposis and surgical management
}

\author{
Md. Monjurul Alam¹, Md. Abu Yusuf Fakir², M. Alamgir Chowdhury ${ }^{3}$
}

\begin{abstract}
Nasal polyps are common, affecting up to four percent of the population. Their etiology remains unclear, but they are known to have associations with allergy, asthma, infection ,cystic fibrosis, and aspirin sensitivity. Computerized tomography allows evaluation of the extent of the disease and is essential when surgical treatment is to be considered. There is good evidence for the use of corticosteroids (systemic and topical) both as primary treatment and as postoperative prophylaxis against recurrence. Surgical treatment has been refined significantly over the past twenty years with the advent of endoscopic sinus surgery. Symptomatic improvement is more in Endoscopic sinus surgery (ESS) compared to conventional procedures (including polypectomy, Caldwel-Iuc and intranasal ethmoidectomy). Overall complications following ESS are relatively very few polyp reccurrence are also less (8\%) for ESS compared with14\% for coventional surgery.
\end{abstract}

Keywords: Nasal polyposis, surgical procedures, FESS

\section{Introduction}

Nasal polyposis are benign lesions, round, soft, semitranslucent, pale or yellow glistening structures that originate from any part of the nasal mucosa or paranasal sinuses (although most commonly from the ethmoid or middle meatus regions) ${ }^{1}$.

Nasal Polyposis have uncertain eitiology and may be linked to chronic inflammation, allergy, autonomic nervous system dysfunction and genetic predisposition. ${ }^{2}$

Functional endoscopic sinus surgery (FESS) is a minimally invasive technique that uses an endoscope to improve ventilation and drainage in addition to polyp removal ${ }^{1,3}$.

The extent of surgery varies according to the extent of disease and surgeon's individual practice. This technique has been used for more than a decade in treating sino-nasal conditions. Advantages are claimed over conventional surgery: permitting a better view of the surgical field, a more precise and thorough clearance of the inflammatory change, fewer complications and lower recurrence rate $1^{4}$.

1. Associate Professor, Dept. of Otolaryngology-Head and neck Surgery, BSMMU, Dhaka

2. Registrar, Dept. of Otolaryngology and Head-Neck Surgery, Dhaka Medical College Hospital, Dhaka

3. Professor, Otolaryngology-Head and Neck Surgery, Medical College for Women \& Hospital Uttara Sector1, Dhaka-1230, Bangladesh.

Address of correspondence : Dr. Md. Monjurul Alam, Associate professor, Otolaryngology, Head \& Neck Surgery, BSMMU, Dhaka.

\section{Objectives}

To provide a systematic review of the clinical effectiveness of endoscopic sinus surgery (ESS) for the removal of nalsal polyps.

\section{Methods}

A systematic review of the literature was undertaken. Searches of electronic databases, websites and reference lists were made to identify relevant studies. Comparative studies were included if they were primary research, focused on FESS for the removal of nasal polyps, reported patient relevant outcomes and were published in English. In addition, case series studies were included if they met the above criteria and enrolled more than 50 patient with polyps.

Twenty-three studies were included in the review article. Etiology, prevalence, clinical features, investigations and treatment of Nasal Polyps are discussed in reference to different authers. Mostly surgical management were discussed in details and medical management also discussed in brief.

\section{Etiology of nasal polyposis}

The etiology of NP is unknown. Some theories consider polyps a consequence of conditions which cause chronic inflammation in the nose and nasal sinuses characterized by stromal edema and variable cellular infiltrate. ${ }^{5}$ Studies provide little evidence to support the relationship of allergy with NP found in only $1 \%-2 \%$ of patients with positive skin prick tests. ${ }^{6}$ 
In addition, studies have shown that NP are no more common in atopic individuals. ${ }^{7}$

An association between polyposis and fungal cultures has been established for many years. ${ }^{8}$

There is some evidence for a genetic element to NP. A link has been demonstrated recently between HLAA74 and NP9 (Luxemburger et al 2000), but the current knowledge in this area remains very limited.

Medical conditions commonly associated with polyps include asthma, bronchiectasis, and cystic fibrosis. ${ }^{10}$

\section{Prevalence}

In the general population, the prevalence of NP is considered to be around $4 \% .{ }^{11}$ They predominantly affect adults and usually present in patients older than 20. They are uncommon in children under 10 and may be the presenting feature of cystic fibrosis. There is a 2:1 male to female preponderance ${ }^{11}$.

Up to a third of NP patients have asthma, whereas polyps are only found in $7 \%$ of asthmatics ${ }^{10}$.

\section{Clinical features}

The main presenting symptom of NP is nasal obstruction which is constant but can vary depending on the site and size of the polyps. Sufferers will also frequently complain of watery rhinorrhea and postnasal drip. Anosmia or hyposmia with an ensuing alteration in taste are also characteristic symptoms of NP. ${ }^{11}$

NP are almost invariably bilateral and when unilateral require histological examination to exclude malignancy or other pathology such as inverted papiloma $^{11}$.

\section{Investigations}

Plain X-rays are insensitive and of no value in the diagnosis of NP but they may show opacification of the affected.

Sinuses ${ }^{12}$. A CT scan will show the extent of NP and anatomical variations and is essential if surgical treatment is to be implemented ${ }^{13}$. In unilateral cases of NP, an magnetic resonance imaging scan may aid diagnosis, particularly for investigations of more serious conditions such as neoplasia ${ }^{14}$.

\section{Treatment of NP}

Therapy for NP involves a combination of observation,medical, and surgical treatments depending on individual case assessment.

\section{Medical treatment}

The advent of topically administered corticosteroids has improved the treatment of upper (NP and rhinitis) and lower (asthma, chronic obstructive airways disease) airway disease ${ }^{15}$. Both topical and systemic glucocorticoids may affect the eosinophil function by both directly reducing eosinophil viability and function or indirectly reducing the secretion of chemotactic cytokines by nasal mucosa and polyp epithelial cells ${ }^{16}$.

Systemic steroids are reserved for advanced or refractory cases particularly when allergy is present. Leukotriene receptor antagonists have recently been shown to be effective ${ }^{17}$, but larger scale trials are required to prove their efficacy.

Allergic fungal rhinosinusitis is treated by both topical and systemic antimycotics as an adjunct to sinus surgery. Surgery is considered the first-line therapy particularly for invasive fungal rhinosinusitis ${ }^{18}$.

\section{Surgical treatment}

No single surgical technique has proved to be entirely curative and patients often undergo repeated procedures despite receiving long-term medical therapy. Recurrence is common in severe disease, rate is $5-10 \%{ }^{15}$.

Surgical techniques have been signifi cantly refi ned over the past 20 years with the advent of endoscopic sinus surgery.

$(\text { ESS })^{4}$. With a better understanding of the anatomy of the osteomeatal complex and pathways of mucocilliary clearance, ESS is now the mainstay of treatment for NP.

In the UK, National Health Service Research and Development Health Technology Assessment Programme Evaluation, polyp recurrence was 28\% following endoscopic sinus surgery compared with $35 \%$ following intranasal polypectomy. ${ }^{01}$

Many surgeons continue to perform simple avulsion polypectomy with good results ${ }^{19}$. ESS involves restoring sinus drainage by careful removal of NP or other soft tissue obstructing the natural sinus ostia ${ }^{2}$. The increasingly more common technique of microdebridement, which involves a rotating blade in combination with suction and irrigation. There is some research available that radical endoscopic sinus procedures involving complete fronto-spheno- 
idectomies lead to greater perceived benefits ${ }^{21}$. More extensive surgery can lead to increased risk of operative complications but to date no comparative study exists between radical and limited surgery.

There is little doubt that ESS offers better visualization and scope for precision surgery. The micro-debrider ${ }^{22}$ (kirtane) in particular enables accurate removal of NP whilst preserving normal anatomical structures such as the turbinates.

Serious complications of ESS are rare but the patient must be counseled preoperatively about the potential risks such as loss of vision, damage to the internal carotid artery, and cerebrospinal fl uid leakage after inadvertent trauma to the skull base ${ }^{23}$. It is important postoperatively to regularly douche the nasal cavity with saline to prevent crusting and adhesions.

Topical intranasal steroids are also a routine part of aftersurgery care to prevent recurrence significantly better in different studies, controlled symptomatic improvement that ranged from 78 to $88 \%$ for FESS compared trials reported overall symptomatic improvement that ranged from 78 to $88 \%$ for FESS compared with 43 to $84 \%$ for conventional techniques (including polypectomy, Caldwell-Luc and intranasal ethmoidectomy) ${ }^{01,23}$.

Disease recurrence was $8 \%$ for FESS compared with $14 \%$ for Caldwell-Luc and polyp recurrence was 28\% for endoscopic ethmoidectomy compared with 35\% for polypectomy ${ }^{1,21,23}$.

Revision surgery was reported in one study only and was the same for FESS and Caldwell-Luc procedures. Percentage of overall complications was reported in only one study and was $1.4 \%$ for FESS compared with $0.8 \%$ for conventional procedures ${ }^{1,4.22,23}$ Total complications in the case series ranged from 22.4 to $0.3 \% .^{1,23}$

\section{Conclusions}

We have identified large amounts of data on FESS. The majority of studies report that people's symptoms improvement following FESS has been satisfactory; however, the improvement magnitude was lower than what has been expected Complications following FESS were relatively very few; however, only a small proportion of evidence is comparative.

FESS may offer some advantages in effectiveness over comparative techniques, but there is enormous variation in the results reported and there are methodological limitations. There is a clear need for quality-controlled trials in order to answer questions regarding the effectiveness of FESS.

Fortunately, advantages in the understanding of sinus anatomy and changes with polyposis, along with many available technological advancements, combine to make surgery safer, efficient, and more complete than in the past.

\section{References:}

1. Dalziel K, Stein K, Round A, et al. Systematic review of endoscopic sinus surgery for nasal polyps. Health Technol Assess, 2003; 7: 1-159.

2. Luxenburger W, Posch G, Berghold A, et al. HLA patterns in patients with nasal polyposis. Eur Arch Otorhinolaryngol, 2000; 257:137-9.

3. Ramalingam R, Ramalingam KK. A hand book of endoscopiec sinus surgery, Chennai, India 1998; 1: 6-7.

4. Kennedy DW, Roth M. Functional Endoscopic Sinus Surgery. Otorhinolaryngology: Head and Neck surgery. $15^{\text {th }}$ edn. Willium \& Wilkins, 1996; 173-180.

5. Bateman ND, Fahy C, Woolford TJ. Nasal polyps: still more questions than answers.. $\mathrm{J}$ Laryngol Otol, 2003; 117: 1-9.

6. Settipane GA, Chafee FH. Nasal polyps in asthma and rhinitis. A review of 6037 patients. $J$ Allergy Clin Immunol, 1977; 59:17-21.

7. Jamal A, Maran AGD. Atopy and nasal polyposis. J Laryngol Otol, 1987; 101:355-8.

8. Safi rstein BH. Allergic bronchopulmonary aspergillosis with obstruction of the upper respiratory tract. Chest, 1976; 70:788-90.

9. Settipane GA. 1996. Epidemiology of nasal polyps. Allergy Asthma Proc, 17:231-6.

10. Hedman J, Kaprio J, Poussa T, et al. Prevalence of asthma, aspirin intolerance, nasal polyposis and chronic obstructive pulmonary disease in a population-based study. Int J Epidemiol, 1999; 28:717-22.

11. Drake-Lee AB. 2004. Nasal polyps. Hospital Med, 65:264-7.

12. linuma T, Hirota Y, Kase Y. Radio-opacity of the paranasal sinuses. Conventional views and CT. 
Rhinology, 1994; 32:134-6.

13. Lund V, Mackay IS. Staging in chronic rhinosinusitis. Rhinology, 1993; 31:183-4.

14. Browne JP,Hopkins C,Stack R, et al. Helth releted quality of life after polypectomy with and without additional surgery.Laryngoscope, 2006; 116: $297-302$.

15. Hedman J, Kaprio J, Poussa T, et al. 1999. Prevalence of asthma, aspirin intolerance, nasal polyposis and chronic obstructive pulmonary disease in a population-based study. Int $\mathrm{J}$ Epidemiol, 28:717-22.

16. Roca-Ferrer J, Mullol J, Xaubet A. Effect of topical anti-infl ammatory drugs on epithelial cell-induced eosinophil survival and GM-CSF secretion. Eur Respir J, 1997; 10:1489-95.

17. Kieff DA, Busaba NY. Effi cacy of montelukast in the treatment of nasal polyposis. Ann Otol Rhinol Laryngol, 2005; 114: 941-5.

18. Schubert MS. Medical treatment of allergic fungal sinusitis. Ann Allergy Asthma Immunol, 2000; 85:90-7.

19. Larsen $\mathrm{K}$, Tos M. A long-term follow-up study of nasal polyp patients after simple polypectomies. Eur Arch Otorhinolaryngol, 1997; 1:85-8.

20. Messerklinger $W$. Role of the lateral nasal wall in the pathogenesis, diagnosis and therapy of recurrent and chronic sinusitis. Laryngo Rhinol Otol, 1987, 66:293-9.

21. Jacobsen J, Svendstrup F. Functional endoscopic sinus surgery in chronic sinusitis- $a$ series of 237 consecutively operated patients. Acta Otolaryngol, 2000; 543:158-61.

22. Kirtane MV, Patel H, Muranjan S. Microdebriders in Endoscopic Sinus Surgery. In: Challenges in Otolaryngology, $1^{\text {st }}$ ed. Jaypee Brothers, 2005; 41-42.

23. Stammberger $\mathrm{H}$. Surgical treatment of nasal polyps: past, present, and future. Allergy, 1999; 54:7-11. 